\section{Sarcoidosis presenting as recurrent left laryngeal nerve palsy}

\author{
F A El-Kassimi, M Ashour, \\ R Vijayaraghavan
}

\begin{abstract}
A patient with sarcoidosis presented with hoarseness caused by mediastinal lymph nodes compressing the left recurrent laryngeal nerve. The response to corticosteroids was dramatic and complete.
\end{abstract}

Hoarseness secondary to left recurrent laryngeal nerve palsy in a patient with mediastinal lymphadenopathy is usually due to malignancy. Tuberculosis ${ }^{12}$ and pneumoconiosis ${ }^{3}$ occasionally produce this complication, however, and sarcoidosis has been implicated in two reports. ${ }^{45}$

Medicine

F A El-Kassimi

Department of

Surgery, College of

Medicine, King Saud

University, Riyadh, Saudi Arabia

$M$ Ashour

R Vijayaraghavan

Address for reprint requests: Dr F A El-Kassimi, Medical Department, College of Medicine, PO Box 2925

Riyadh 11461, Saudi Arabia

Accepted 23 January 1990

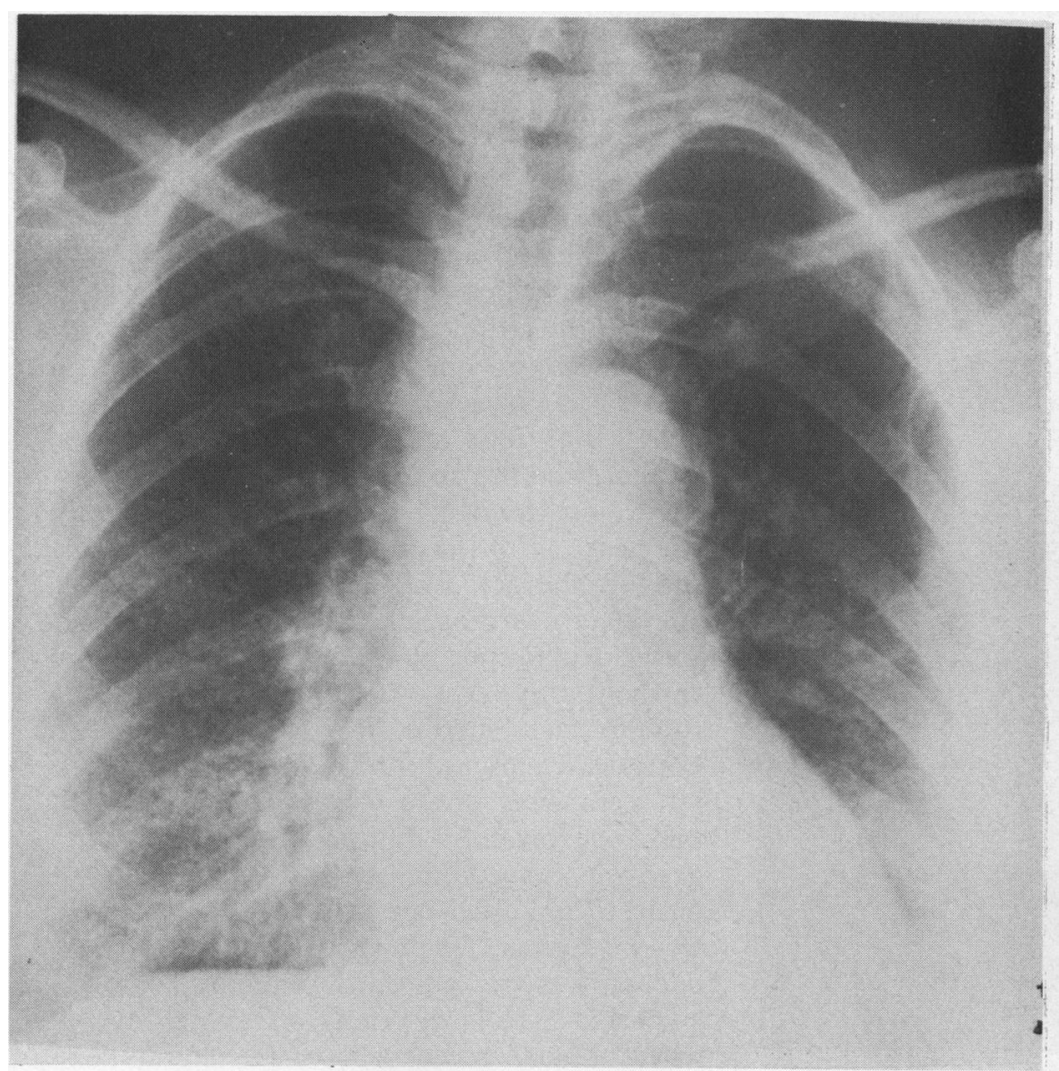

Figure 1 Chest radiograph at presentation showing interstitial infiltrate in the lower and middle zones with widening of the mediastinum. for the hoarseness. Laryngoscopy showed paralysis of the left vocal cords with no intrinsic lesion of the larynx. The palate moved normally and the gag reflex was present bilaterally.

The full blood count was normal and her erythrocyte sedimentation rate was $10 \mathrm{~mm}$ in the first hour. Chest radiography (fig 1) showed bilateral interstitial infiltrate in the lower and middle zones of the lung, with widening of the mediastinum and probable hilar lymphadenopathy. Computed tomography of the lung confirmed the presence of interstitial infiltrate in the lung as well as paratracheal, carinal, and hilar lymphadenopathy (fig 2). The Mantoux test (10 units tuberculin) produced a negative response on two occasions. Results of liver function tests and the serum calcium concentration were normal. Arterial blood gases sampled at rest were normal, as were spirometric values and total lung capacity; carbon monoxide transfer factor was substantially reduced, however, at $39 \%$ predicted (transfer coefficient $40 \%$ predicted). A gallium scan showed increased uptake in hilar nodes, mediastinum, lungs, and parotid glands.

A paratracheal lymph node biopsy specimen, obtained by mediastonoscopy, showed non-caseating grauloma with no acid fast bacilli or fungi, and no mycobacteria or fungi were grown in culture. A diagnosis of sarcoidosis was made and prednisolone $40 \mathrm{mg}$ daily was started. Ten days later the hoarseness and nerve palsy had cleared and chest radiography showed total resolution of both interstitial and nodal lesions.

The patient has remained symptomless with a normal chest radiograph for six months after presentation.

\section{Discussion}

Hoarseness in association with mediastinal lymphadenopathy is usually due to malignant disease. As this case shows, sarcoidosis should also be considered, particularly in patients who are generally well or who have concomitant interstitial lung disease. Hoarseness in sarcoidosis may be caused by three

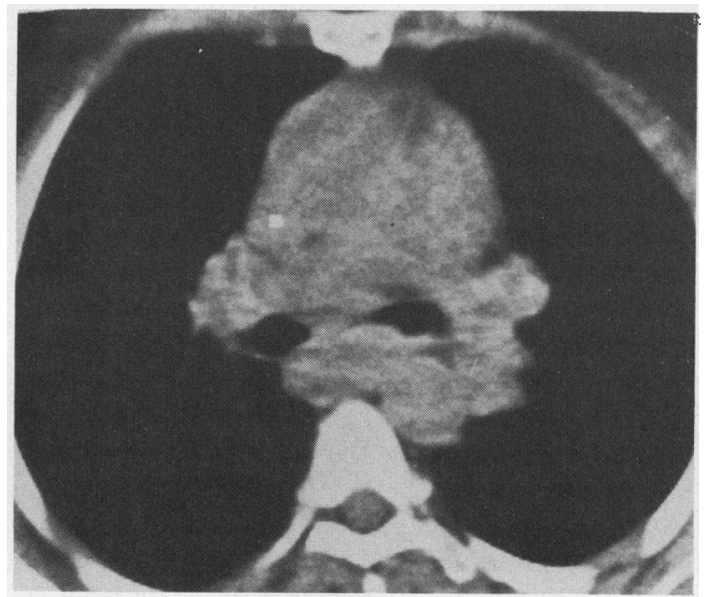

Figure 2 Computed tomogram at presentation showing bilateral hilar lymphadenopathy. 
mechanisms: (1) granulomatous infiltration of the larynx with normal vocal cord motility, occurring in $5 \%$ of cases 6 -a serious lesion that might result in stridor and respiratory distress; (2) sarcoid cranial polyneuropathy with dysphonia and dysphagia, resulting from sarcoid affecting the vagus nerve-a condition invariably associated with other neurological lesions, particularly facial nerve palsy ${ }^{7}$; (3) compression of the left recurrent laryngeal nerve,${ }^{45}$ produced by a sarcoid lymph node as it hooks around the arch of the aorta, as in carcinoma of the bronchus.

To our knowledge, compression of the left recurrent laryngeal nerve by sarcoid nodes has been described on only two previous occasions. ${ }^{45}$ Other benign conditions causing mediastinal lymphadenopathy and compression of the left recurrent laryngeal nerve- namely, tuberculosis ${ }^{12}$ and pneumoconiosis ${ }^{3}-$ have been described rarely.

1 Fowler RW, Hetzel MR. Tuberculous mediastinal lymphadenopathy can cause left vocal cord paralysis. $\mathrm{Br}$ Med 1983;286:1562.

2 Hamilton JRL, Varghese G, Shepperd HWH, Stevenson HM. Tuberculous left vocal cord palsy: a timely reminder. $J$ Laryngol Otol 1986;100:837-8.

3 Sherani TM, Angelini GD, Passani SP, Butchart EG. Vocal cord paralysis associated with coalworkers' pneumoconiosis and progressive massive fibrosis. Thorax 1984; 39:683-4.

4 Swinburn CR, Pozniak AL, Davies DG, Treasure T, Johnson NM. Left recurrent laryngeal nerve palsy as the presenting feature of sarcoidosis. Sarcoidosis 1986;31: presentin

5 Chijimatsu Y, Tajima J, Washizaki M, Homma H. Hoarseness as an initial manifestation of sarcoidosis. Ches 1980;78:779-81.

6 Neville E. Upper respiratory tract sarcoidosis. Semin Respir Med 1986;8:52-8.

7 Matthews WB. Sarcoid neuropathy. In: Dyck PJ, Thomas PK, Lambert EH, Bunce R, eds. Peripheral neuropathy. Vol 2. 2nd ed. Philadelphia: Saunders, 1984:2018-20.

Thorax 1990;45:566-567

\section{Constrictive pericarditis caused by primary liposarcoma}

\author{
R J Lionarons, J van Baarlen, \\ J F Hitch cock
}

\section{Department of}

\section{Cardiology}

R J Lionarons

\section{Department of}

Pathology

$\mathrm{J}$ van Baarlen

Department of

Cardiothoracic

Surgery

J F Hitchcock

University Hospital,

Utrecht,

The Netherlands

Address for reprint requests: Dr R J Lionarons,

Department of Cardiology,

Militair Hospitaal

"Dr A Mathijsen",

J Haydnlaan 2, $3533 \mathrm{AE}$

Utrecht, The Netherlands.

Accepted 18 January 1990

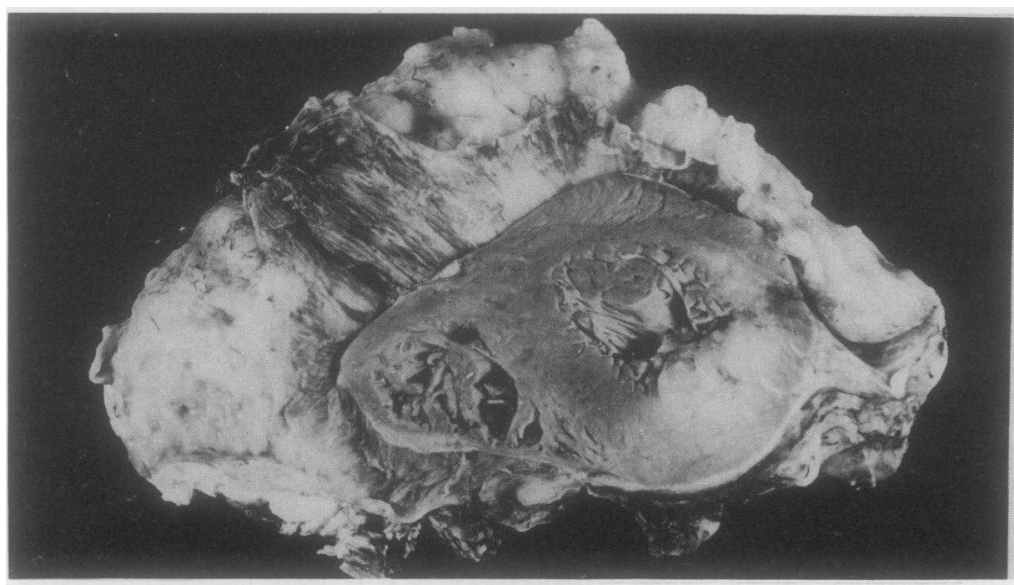

Figure 1 Transverse section of the heart at the level of both ventricles showing the tumour extending from the parietal to the visceral pericardium. At the lower right side the tumour has infiltrated the left ventricular wall. constrictive pericarditis caused by primary liposarcoma in a 30 year old man.

\section{Case report}

A 30 year old white man presented with a two month history of progressive dyspnoea on exertion, left sided chest pain that radiated to the left shoulder, general malaise, weight loss, anorexia, night sweats, fever, and pronounced peripheral oedema. He was transferred to us from a small regional hospital with a diagnosis of suspected right sided endocarditis. On examination the patient was ill with severe dyspnoea at rest. His blood pressure was 120/ $85 \mathrm{~mm} \mathrm{Hg}$ with pulsus paradoxus of about 30 $\mathrm{mm} \mathrm{Hg}$. The heart rate was regular at 116 beats $/ \mathrm{min}$ and the temperature was $37 \cdot 8^{\circ} \mathrm{C}$. Venous pressure was visible at the angle of the jaw and his abdomen was swollen and tender with considerable hepatomegaly and ascites. Severe pitting oedema of the legs extended to the sacrum. There were broadened precordial pulsations, an apex beat palpated outside the midclavicular line, and very faint heart sounds, with a grade $2 / 6$ systolic murmur along the right sternal border and a pericardial friction rub near the apex.

A chest radiograph showed enlargement of the heart, with irregular margins extending to the left thoracic wall, and a pleural effusion. An electrocardiogram was low voltage and showed non-specific $T$ wave changes. Echocardiography showed a huge, irregular tumour mass surrounding the greater part of both ventricles and infiltrating the apex of the left ventricle. There was considerable limitation of diastolic filling. Computed tomography showed no extension outside the pericardium. An anterolateral thoracotomy was performed for diagnostic purposes and to decompress the myocardium. The heart was encircled by a large, richly vascular, mucoid, multilobular, green-yellow, glistening mass, encapsulated by the pericardium and attached to the apex of the left ventricle. 